\title{
Liraglutide ameliorates renal injury in streptozotocin-induced diabetic rats by activating endothelial nitric oxide synthase activity via the downregulation of the nuclear factor- $\kappa B$ pathway
}

\author{
SAI-JUN ZHOU ${ }^{1 *}$, LIAN BAI $^{1,2^{*}}$, LIN LV $^{1}$, RUI CHEN $^{1}$, CHUN-JUN LI $^{1}$, \\ XIANG-YANG LIU ${ }^{1}$, DE-MIN YU ${ }^{1}$ and PEI YU ${ }^{1}$
}

\begin{abstract}
${ }^{1}$ Department of Diabetic Nephropathy Hemodialysis, 2011 Collaborative Innovation Center of Tianjin for Medical Epigenetics,
Key Laboratory of Hormones and Development (Ministry of Health), Metabolic Diseases Hospital \&

Tianjin Institute of Endocrinology, Tianjin Medical University, Tianjin 300070; ${ }^{2}$ Department of Endocrinology,

Dongying Municipal Authorities Hospital, Dongying, Shandong 257091, P.R. China
\end{abstract}

Received December 8, 2013; Accepted January 1, 2014

DOI: $10.3892 / \mathrm{mmr} .2014 .2555$

\begin{abstract}
Accumulating evidence has implicated that liraglutide, one of the human glucagon-like peptide-1 (GLP-1) analogues, elicits protective effects on diabetic nephropathy; however, the mechanism has yet to be fully elucidated. The present study aimed to assess the effect and underlying mechanisms of liraglutide in diabetic nephropathy. Wistar rats with streptozotocin-induced diabetes mellitus were subcutaneously injected with liraglutide or phosphate buffer for 12 weeks at a dose of $0.3 \mathrm{mg} / \mathrm{kg} / 12 \mathrm{~h}$. The biochemical parameters were determined, renal histological examination was performed by hematoxylin and eosin and periodic acid Schiff base staining, and the mRNA levels of nuclear factor $\kappa \mathrm{B}(\mathrm{NF}-\kappa \mathrm{B})$ and endothelial nitric oxide synthase (eNOS) were assessed by quantitative polymerase chain reaction. Furthermore, the protein expression of NF- $\kappa \mathrm{B}$ and eNOS as well as eNOS phosphorylation were examined by western blot analysis and the levels of inflammatory cytokines downstream of $\mathrm{NF}-\kappa \mathrm{B}$ were evaluated by fluorescence-assisted cell sorting and finally, the eNOS activity and nitric oxide (NO) production were evaluated by ELISA. Liraglutide decreased the levels of total cholesterol, urine, 24-h urinary albumin,
\end{abstract}

Correspondence to: Professor De-min Yu or Professor Pei Yu, Department of Diabetic Nephropathy Hemodialysis, 2011 Collaborative Innovation Center of Tianjin for Medical Epigenetics, Key Laboratory of Hormones and Development (Ministry of Health), Metabolic Diseases Hospital \& Tianjin Institute of Endocrinology, Tianjin Medical University, 66 Tongan Street, Heping, Tianjin 300070, P.R. China

E-mail: deminyu@126.com

E-mail: peisyu@yeah.net

*Contributed equally

Key words: diabetic nephropathy, endothelial nitric oxide synthase, nuclear factor- $\kappa \mathrm{B}$, inflammatory cytokine blood urea nitrogen, serum creatinine and histological damage. Liraglutide also reduced the expression of NF- $\mathrm{KB}$ at mRNA and protein levels; the expression of tumor necrosis factor- $\alpha$, interferon- $\gamma$, interleukin- 6 and monocyte chemoattractant protein-1 were also reduced. By contrast, eNOS phosphorylation, eNOS activity and NO production appeared to have increased. Liraglutide may have a direct beneficial effect on diabetic nephropathy by improving eNOS activity by inhibiting the NF- $\mathrm{BB}$ pathway without eliciting a glucose lowering effect.

\section{Introduction}

Diabetic nephropathy is one of the leading causes of end-stage renal failure (1). The inactivation of endothelial nitric oxide synthase (eNOS) has been considered to be a recently discovered mechanism of diabetic nephropathy $(2,3)$, which severely impairs glomerular endothelial cell nitric oxide (NO) production and causes glomerular endothelial cell dysfunction, which are impairments that contribute to the development of diabetic nephropathy $(2,4)$. However, the specific mechanism that causes eNOS inactivation remains elusive.

The nuclear factor (NF)- $\mathrm{BB}$ signaling pathway is a major pathway of chronic inflammation. Previously, it was demonstrated that the NF- $\kappa \mathrm{B}$ signaling pathway regulates the eNOS activity of aortic endothelial cells (5). Studies have also revealed that NF- $\mathrm{KB}$ signaling pathways in the diabetic kidney are abnormally activated and NF- $\mathrm{KB}$ signaling pathway inhibitors may significantly reduce the damage to the kidneys in diabetic rats $(6,7)$. Therefore, the NF- $\mathrm{KB}$ signaling pathway may participate in the development of diabetic nephropathy by regulating the eNOS activity of glomerular endothelial cells.

Human glucagon-like peptide-1 (GLP-1) analogues, such as liraglutide, have been demonstrated to elicit protective effects in diabetic vascular complications (4), thus suggesting that liraglutide exhibits an endothelial cell function. Other investigations have also demonstrated that the protective effect 
of liraglutide on vascular systems involves the reduction of chronic inflammation $(7,8)$. These studies have indicated that liraglutide may be involved in eNOS activity by alleviating chronic inflammation.

In the present study, the effect of liraglutide on diabetic nephropathy was examined. The correlation between eNOS activity and the NF- $\mathrm{NB}$ chronic inflammation pathway was also determined in rats with diabetic nephropathy.

\section{Materials and methods}

Animals. A total of 45 seven-week-old male Wistar rats were purchased from Tianjin Radiology Institution (Tianjin, China) and provided with a standard diet and water. The animals were kept under natural circadian light/dark cycles under conditions of $18-22^{\circ} \mathrm{C}$ and $40-70 \%$ humidity. This study was conducted in strict accordance with the recommendations in the Guide for the Care and Use of Laboratory Animals of the National Institutes of Health. The animal use protocol was reviewed and approved by the Institutional Animal Care and Use Committee (IACUC) of Tianjin Medical University. Diabetes was induced by injecting streptozotocin (STZ; Sigma-Aldrich, St. Louis, MO, USA) in $0.1 \mathrm{~mol} / 1$ citrate buffer $\mathrm{pH} 4.5$ at a dose of $45 \mathrm{mg} / \mathrm{kg}$ body weight. The rats that fasted but revealed blood glucose levels $>16.7 \mathrm{mmol} / 1$ were considered diabetic. The rats injected with citrate buffer alone were used as non-diabetic control samples. One week following the induction of diabetes, half of the diabetic rats were randomly selected and subcutaneously injected with the GLP-1 analogue liraglutide (Novo Nordisk Co, Copenhagen, Denmark) at a dose of $0.3 \mathrm{mg} / \mathrm{kg} / 12 \mathrm{~h}$ for 12 weeks as previously reported $(9,10)$. The remaining diabetic rats were injected with phosphate buffer and used as the control sample. The rats were housed in individual metabolic cages for the 24-h urine collection on the day prior to the administration of STZ and at the end of 12-week treatment. Urine was centrifuged at $800 \mathrm{x} \mathrm{g}$ for $10 \mathrm{~min}$ at room temperature. Whole urine was stored at $-80^{\circ} \mathrm{C}$ until use. At the end of the 12 -week treatment, the rats were sacrificed and blood was collected from the left ventricle. Blood glucose (BG), total cholesterol (TC), triglycerides (TG), serum creatinine (Scr) and blood urea nitrogen (BUN) as well as microalbuminuria were determined using an automatic biochemical analyzer (7070; Hitachi Ltd., Tokyo, Japan). The kidneys were immediately excised and stored at $-80^{\circ} \mathrm{C}$ until use.

Histopathological examination. The rats from each group were sacrificed at the end of the 12 -week treatment by anaesthetising them with diethyl ether. The kidneys were excised to evaluate the histopathological changes. The procedures were performed as described previously (11). Briefly, the kidneys were carefully excised and then washed with phosphate-buffered saline (PBS). Following this, the kidneys were stored in $10 \%$ neutral buffered formalin and embedded in paraffin. Four micron sections were cut and stained with hematoxylin and eosin (H\&E; Tianjin Runtai, Co., Ltd., Tianjin, China) and periodic acid Schiff base (PAS; Tianjin Runtai, Co., Ltd.) for histopathological observations using a HMIAS-2000 Image Analysis system (Guangzhou Longest Technology, Guangzhou, China). The sections were then analyzed for the degree of tubular and glomerular damage. Glomerular damage index (GDI) was calculated from 0 to 4 on the basis of the degree of glomerulosclerosis, mesangiolysis and mesangial expansion. Approximately 80-100 glomerular cells from the renal cortex were observed for each section. GDI was obtained by averaging the scores from the counted glomerular cells.

Determination of inflammatory cytokine levels in rat kidneys. Renal tissue was homogenized in $10 \mathrm{mM}$ Tris- $\mathrm{HCl}$ buffer solution ( $\mathrm{pH}$ 7.4) containing $2 \mathrm{M} \mathrm{NaCl}, 1 \mathrm{mM}$ EDTA, $0.01 \%$ Tween-80 and $1 \mathrm{mM}$ phenylmethanesulfonyl fluoride (PMSF), and centrifuged at $9,000 \mathrm{x}$ g for $30 \mathrm{~min}$ at $4^{\circ} \mathrm{C}$. The resulting supernatant was used for cytokine determination. A Cytometric Bead array (CBA; BD Biosciences, Franklin Lakes, NJ, USA) mouse inflammation kit was used to determine the levels of interleukin (IL)-6, IL-10, IL-12p70, interferon- $\gamma$ (IFN- $\gamma)$, tumor necrosis factor- $\alpha$ (TNF- $\alpha$ ) and monocyte chemotactic protein 1 (MCP-1) according to the manufacturer's instructions (12). The data were acquired on a FACSCalibur flow cytometer (BD Biosciences) and analyzed using FCAP Array software version 3.0 (BD Biosciences).

Quantitative polymerase chain reaction ( $q P C R)$. Total RNA from rat kidneys was extracted using TRIzol reagent (Life Technologies Co., Carlsbad, CA, USA) according to the manufacturer's instructions. Approximately $2 \mu \mathrm{g}$ of RNA was reverse transcribed into cDNA by using Moloney murine leukemia virus reverse transcriptase (Invitrogen Life Technologies). The primers were designed using Primer 5.0 software (Premier Biosoft International, Palo Alto, CA, USA) and primers were as follows: $\mathrm{NF}-\kappa \mathrm{B}$, forward 5'-GTATGGCTTCCCGCACTATGG-3' and reverse 5'-TCGTCACTCTTGGCACAATCTC-3'; eNOS forward 5'-GACATTGAGAGCAAAGGGCTGC-3' and reverse 5'-CGGCTTGTCACCTCCTGG-3'; $\beta$-actin forward 5'-TGGAGAAGAGCTATGAGCTGCCTG-3' and reverse 5'-GTGCCACCAGACAGCACTGTGTTG-3'. The reaction conditions were set as follows: $95^{\circ} \mathrm{C}$ for $2 \mathrm{~min}, 40$ cycles at $95^{\circ} \mathrm{C}$ for $30 \mathrm{sec}$, then $56^{\circ} \mathrm{C}$ for $30 \mathrm{sec}$ and $72^{\circ} \mathrm{C}$ for $30 \mathrm{sec}$. Melting curve analysis was performed from $55-95^{\circ} \mathrm{C}$ by monitoring fluorescence at a $0.5^{\circ} \mathrm{C}$ increase with $30 \mathrm{sec}$ intervals. The sample measurements were performed in triplicate. For each experimental sample, the target mRNA levels were normalized to $\beta$-actin mRNA.

Western blot analysis. Total protein from rat kidneys was prepared by lysing buffer containing protease inhibitors (20 mM Tris, pH 7.4; $150 \mathrm{mM} \mathrm{NaCl} ; 1 \mathrm{mM}$ EDTA; $1 \mathrm{mM}$ PMSF; $1 \mathrm{mM}$ orthovanadate; $1 \mu \mathrm{g} / \mathrm{ml}$ leupeptin; $10 \mu \mathrm{g} / \mathrm{ml}$ aprotinin). Following homogenization and centrifugation, the amount of protein in the supernatant was determined with a bicinchoninic acid protein assay kit (Pierce Biotechnology, Inc., Chicago, IL, USA). The aliquots of extract (35 $\mu \mathrm{g}$ of protein/lane) from each sample were loaded on SDS-PAGE using a $10 \%$ Tris-glycine gel, and then transferred onto a polyvinylidene difluoride (PVDF) membrane at $30 \mathrm{~V}$ for $2 \mathrm{~h}$. The membrane was blocked for $1 \mathrm{~h}$ at room temperature with $5 \%$ skimmed milk and incubated for $1 \mathrm{~h}$ at room temperature with the following primary antibodies: Anti-NF- $\kappa \mathrm{B}$ (Cell Signaling Technology, Inc., Danvers, MA, USA), anti-p65 (Santa Cruz 

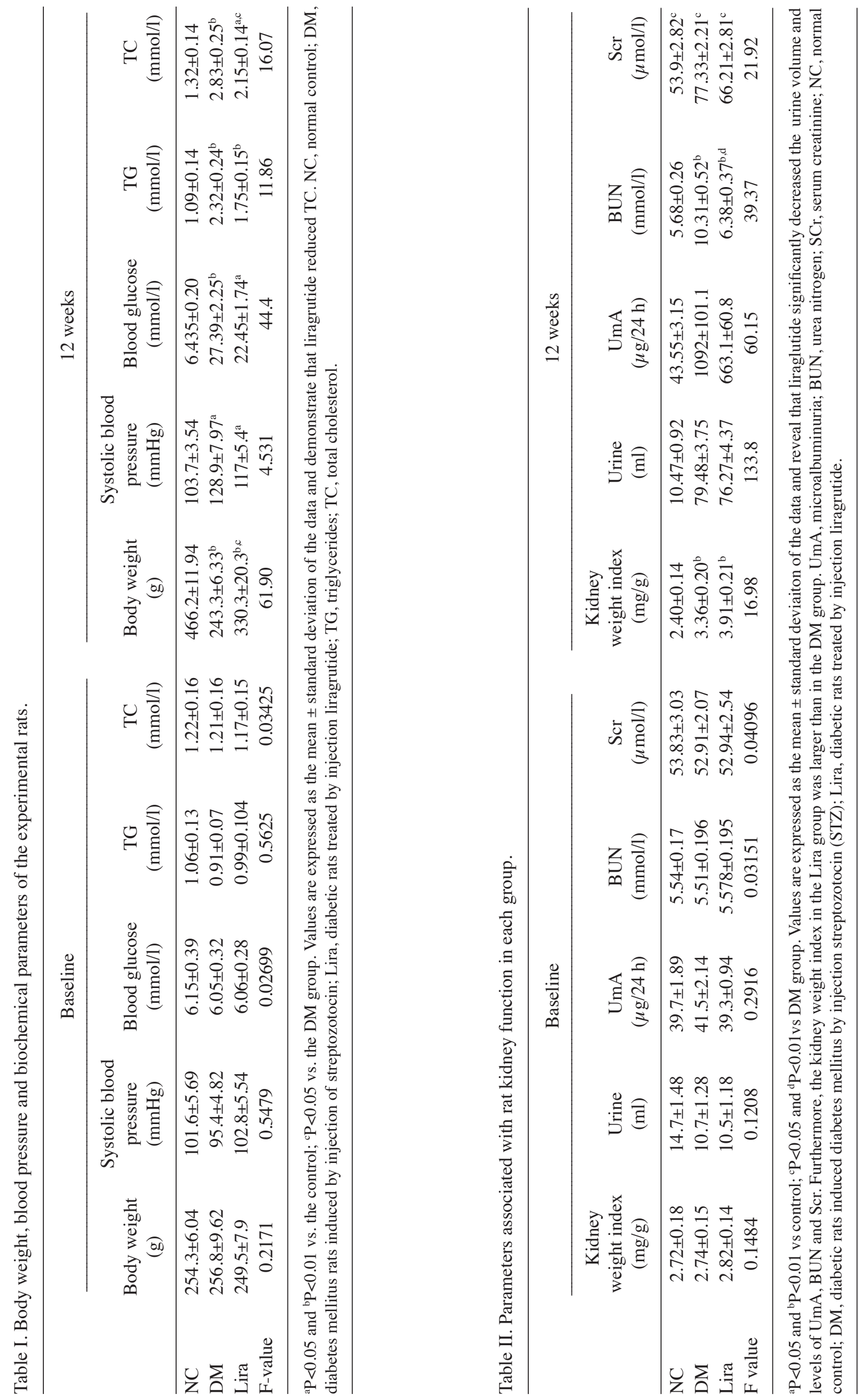

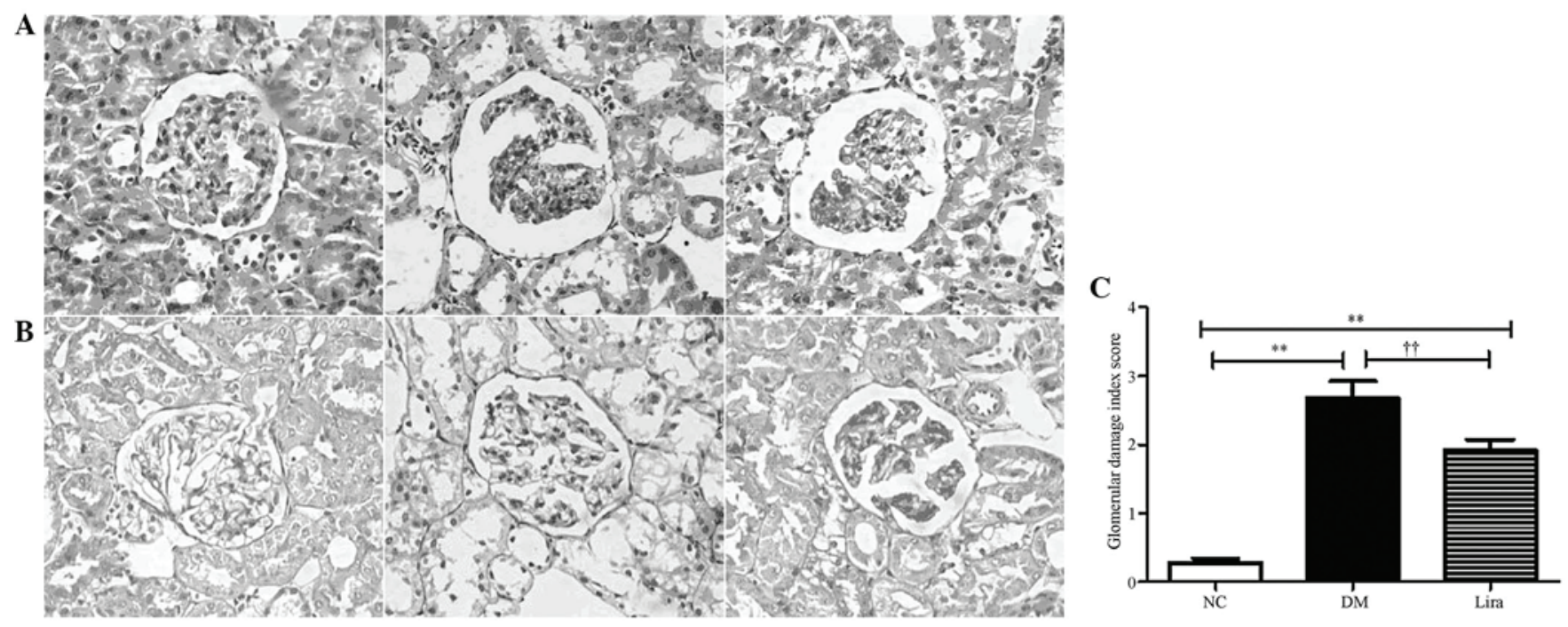

Figure 1. Liragrutide ameliorates histological damage of kidneys in diabetic rats. Representative (A) hematoxylin and eosin staining and (B) PAS staining for histopathological observations of the kidney sections from each group of rats. To semiquantify the damage of kidneys, the GDI was determined. (C) The GDI was calculated for the PAS-stained sections at a magnification of $x 400$ with a score system of 0 to $4: 0$ represents no lesion; $1+$ represents sclerosis $<25 \%$; $2+$ represents sclerosis of $25-50 \%$ of the glomerus; $3+$ represents sclerosis of $50-75 \%$ of the glomerus; $4+$ represents sclerosis $>75 \%$. Values are presented as the mean \pm standard deviation $(\mathrm{n}=6) .{ }^{* *} \mathrm{P}<0.01$ vs. the control; ${ }^{\dagger} \mathrm{P}<0.01$ vs. the DM group. NC, normal control; DM, diabetes mellitus rats induced by injection of streptozotocin; Lira, diabetic rats treated by injection of liragrutide; GDI, glomerular damage index; PAS, periodic acid Schiff base.

Biotechnology, Inc., Santa Cruz, CA, USA), anti-p-eNOS (Ser 1177; Cell Signaling Technology, Inc.) and anti-eNOS (Cell Signaling Technology, Inc.). The membranes were washed and then the secondary antibody was added and incubated for $1 \mathrm{~h}$ at room temperature. Peroxidase activity on the PVDF membranes was visualized on an X-ray film with an ultraviolet transmission analyzer. Protein levels were normalized to $\beta$-actin.

Measurement of glomerular eNOS activity and NO levels. An eNOS activity classifying kit and an NO test kit (Nanjing Jiancheng Bioengineering Institute, Nanjing, China) were used to detect the eNOS and NO levels in the glomeruli, respectively. The procedures were performed as described previously (4).

Statistical analysis. Statistical analyses were conducted using SPSS 15.0 software (SPSS, Inc., Chicago, IL, USA). All values are expressed as the mean \pm standard deviation. The significance of differences among the experimental groups was determined by analysis of variance. $\mathrm{P}<0.05$ was considered to indicate a statistically significant difference.

\section{Results}

Rats' body weights, blood pressure and biochemical parameters. Rats' body weights, blood pressure and biochemical parameters were evaluated prior to and following 12 weeks of administration of liraglutide $(0.3 \mathrm{mg} / \mathrm{kg} / 12 \mathrm{~h})$. The body weight, blood pressure and biochemical parameters of rats are summarized in Table I. No differences were observed in body weight, systolic blood pressure, BG, TG and TC in each group at baseline $(\mathrm{P}>0.05)$. Following liraglutide treatment for 12 weeks, systolic blood pressure, TG and TC of both diabetic groups were significantly higher than those of the normal control group. Liraglutide was able to significantly reduce TC $(\mathrm{P}<0.05)$, and may decrease systolic blood pressure $(\mathrm{P}=0.089)$ and TG $(\mathrm{P}=0.065)$. Compared with the NC group, the body weights were decreased in the diabetes and liraglutide groups $(\mathrm{P}<0.01)$, while being increased in liraglutide treatment group as compared with the DM group $(\mathrm{P}<0.05)$. No significant difference was observed in the BG between the DM and liraglutide groups $(\mathrm{P}>0.05)$.

Parameters associated with the kidney function of rats. The parameters associated with kidney function, including kidney weight index, urine volume, microalbuminuria (UmA), BUN and Scr, were examined prior to and following liraglutide treatment for 12 weeks. No significant difference was observed in all of the parameters in each group at the baseline. Kidney weight index, urine volume, UmA, BUN and Scr significantly increased in the diabetic rats with or without liraglutide compared with those of the normal control rats $(\mathrm{P}<0.05$ or $\mathrm{P}<0.01)$. Liraglutide significantly decreased the urine volume and levels of UmA, BUN and Scr. Kidney weight index in the liraglutide group was higher than that in the DM group (Table II).

Histological changes in diabetic rat kidneys. Following liraglutide was administered for 12 weeks, the histological changes in the kidneys were evaluated by H\&E and PAS staining, respectively. Fig. 1 illustrates the changes in the kidney of the rats in all of the groups. The diabetic rats demonstrated several changes, including inflammatory cell infiltration, mesangial expansion and proliferation in the glomeruli. Prominent nodular glomerulosclerosis with glomerular basement membrane thickening was also observed. However, minimal changes were observed in the diabetic rats that were supplemented with liraglutide. The semiquantitative analysis demonstrated that liraglutide significantly decreased the GDI of diabetic rats. 

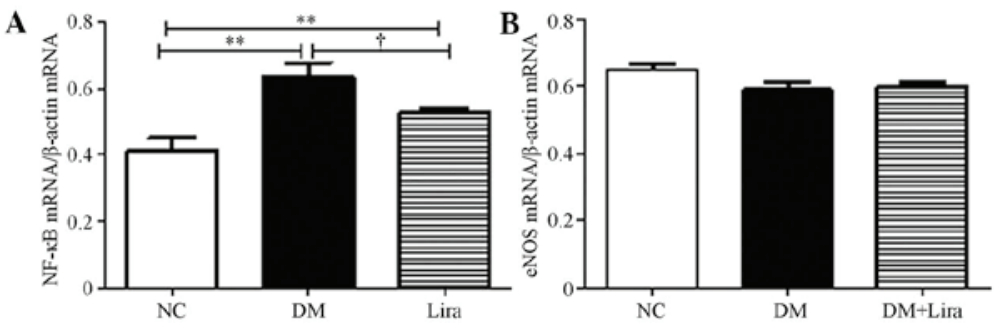

Figure 2. Liraglutide reduces the expression of NF- $\mathrm{B}$ mRNA but not eNOS mRNA in diabetic kidneys. The effects of liraglutide on renal (A) NF- $\mathrm{BB}$ and (B) eNOS mRNA expression. The relative expression levels of eNOS mRNA in the rat glomerular homogenates were assessed by semi-quantitative polymerase chain reaction. The mean value of mRNA levels was expressed as ratios relative to that of $\beta$-actin. The results are expressed as the mean \pm standard deviation. ${ }^{*} \mathrm{P}<0.05$ and ${ }^{* *} \mathrm{P}<0.01$ vs. the control; ${ }^{\circ} \mathrm{P}<0.05$ vs. the DM group. DM, diabetes mellitus rats induced by injection of streptozotocin; Lira, diabetic rats treated with an injection liragrutide; NF- $\mathrm{B}$, nuclear factor-kappa B; eNOS, endothelial nitric oxide synthase.
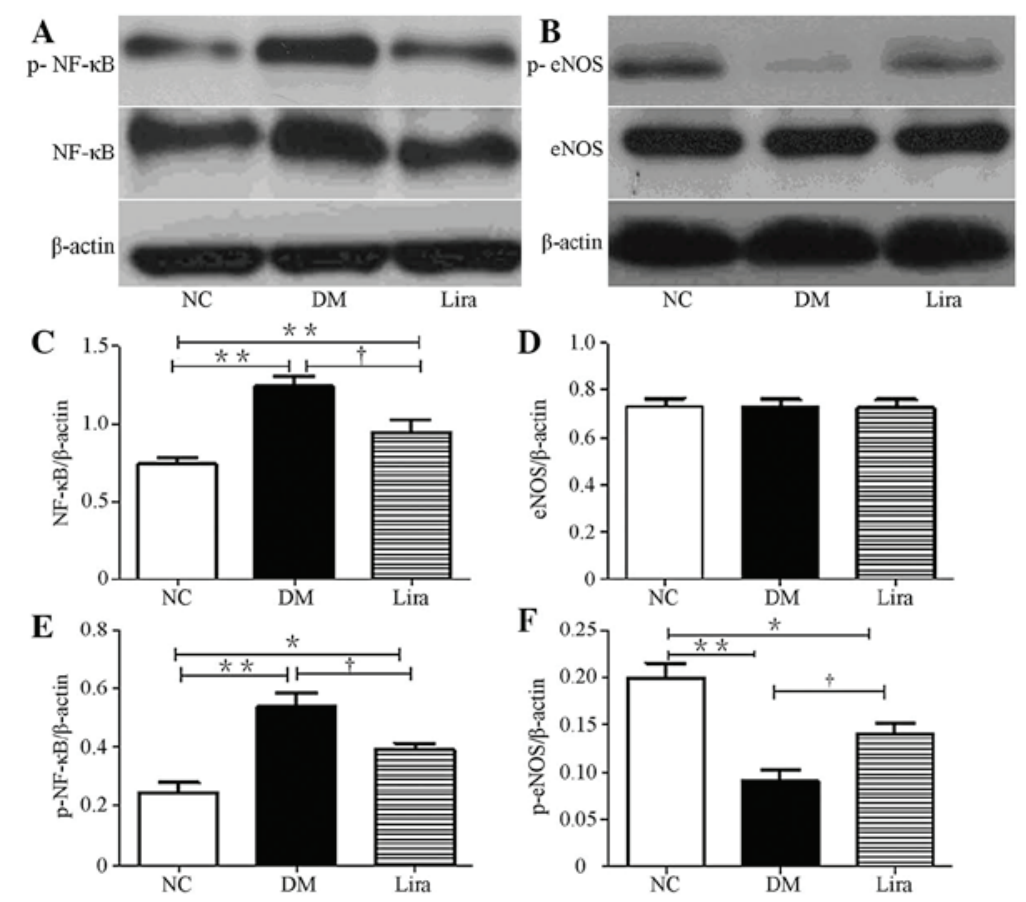

Figure 3. Effect of liraglutide on the expression of NF- $\kappa B$ and eNOS in diabetic rats. The effects of liraglutide on renal (A) NF- $\kappa B$ (p65), p-NF- $\mathrm{kB}$ (Ser 276) expres-

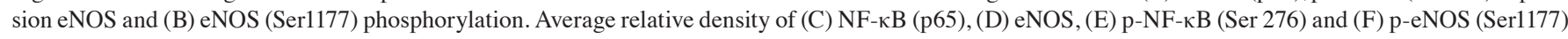
relative to $\beta$-actin from the four separate experiments. The results are presented as the relative density of the controls. Data are expressed as the mean \pm standard error of the mean. ${ }^{* *} \mathrm{P}<0.01$ vs. the control; ${ }^{\circ} \mathrm{P}<0.05$ and ${ }^{\dagger} \mathrm{P}<0.01$ vs. DM group. DM, diabetes mellitus rats induced by injection of streptozotocin; Lira, diabetic rats treated by injection liragrutide; NF- $\mathrm{B}$, nuclear factor-kappa B; eNOS, endothelial nitric oxide synthase.

Effects of liraglutide on $N F-\kappa B(p 65)$ and eNOS expression. The mRNA expression of NF- $\kappa B$ and eNOS were measured following the 12-week treatment with or without liraglutide. The results demonstrated that the mRNA levels in diabetic kidneys increased $(\mathrm{P}<0.01)$. Liraglutide inhibited this increase $(\mathrm{P}<0.05$; Fig. 2A). No significant difference was observed in the mRNA levels of eNOS between each group $(\mathrm{P}>0.05$; Fig. 2B).

The effects of liraglutide on the protein levels of NF- $\mathrm{KB}$ and eNOS in the diabetic rat kidneys was also examined. The expression levels of NF- $\kappa \mathrm{B}$ and $\mathrm{p}-\mathrm{NF}-\kappa \mathrm{B}(\mathrm{p} 65)$ in diabetic groups were higher than those in the normal control group $(P<0.01)$. Liraglutide reduced this increase $(\mathrm{P}<0.01$; Figs. 3A, $\mathrm{C}$ and $\mathrm{E})$. No significant difference was observed between each group in terms of the expression levels of eNOS, but eNOS (ser 1177) phosphorylation was reduced in both diabetic groups (Figs. 3B and D). Liraglutide significantly restored eNOS (ser 1177) phosphorylation (Figs. 3B, D and F).

Effect of liraglutide on the levels of inflammatory cytokines. $\mathrm{NF}-\kappa \mathrm{B}$ could regulate inflammatory cytokine expression. In the present study, the levels of inflammatory cytokines of diabetic kidneys were further examined using the CBA flow cytometry kit. The results demonstrated that TFN- $\alpha$, IFN- $\gamma$, IL-6 and MCP-1 increased significantly in the diabetic kidneys $(\mathrm{P}<0.01$ or $\mathrm{P}<0.05)$ and liraglutide was able to inhibit these effects $(\mathrm{P}<0.01$ or $\mathrm{P}<0.05$; Figs. $4 \mathrm{~A}, \mathrm{~B}, \mathrm{D}$ and $\mathrm{F})$. A decrease in IL-10, which is an anti-inflammatory cytokine in diabetic kidneys, was noted. An increasing trend was observed in the liraglutide treatment group, but this increase was not statistically significant (Fig. 4C). No significant difference was noted in the levels of IL-12p70 between the groups ( $\mathrm{P}>0.05$; Fig. 4E). 
A

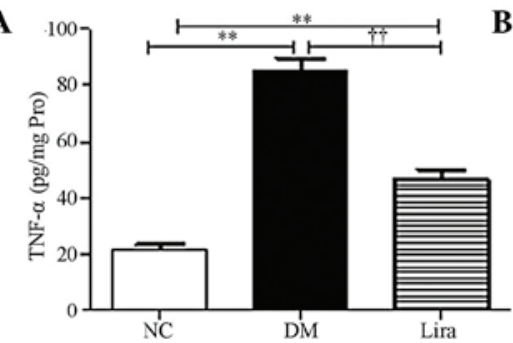

C
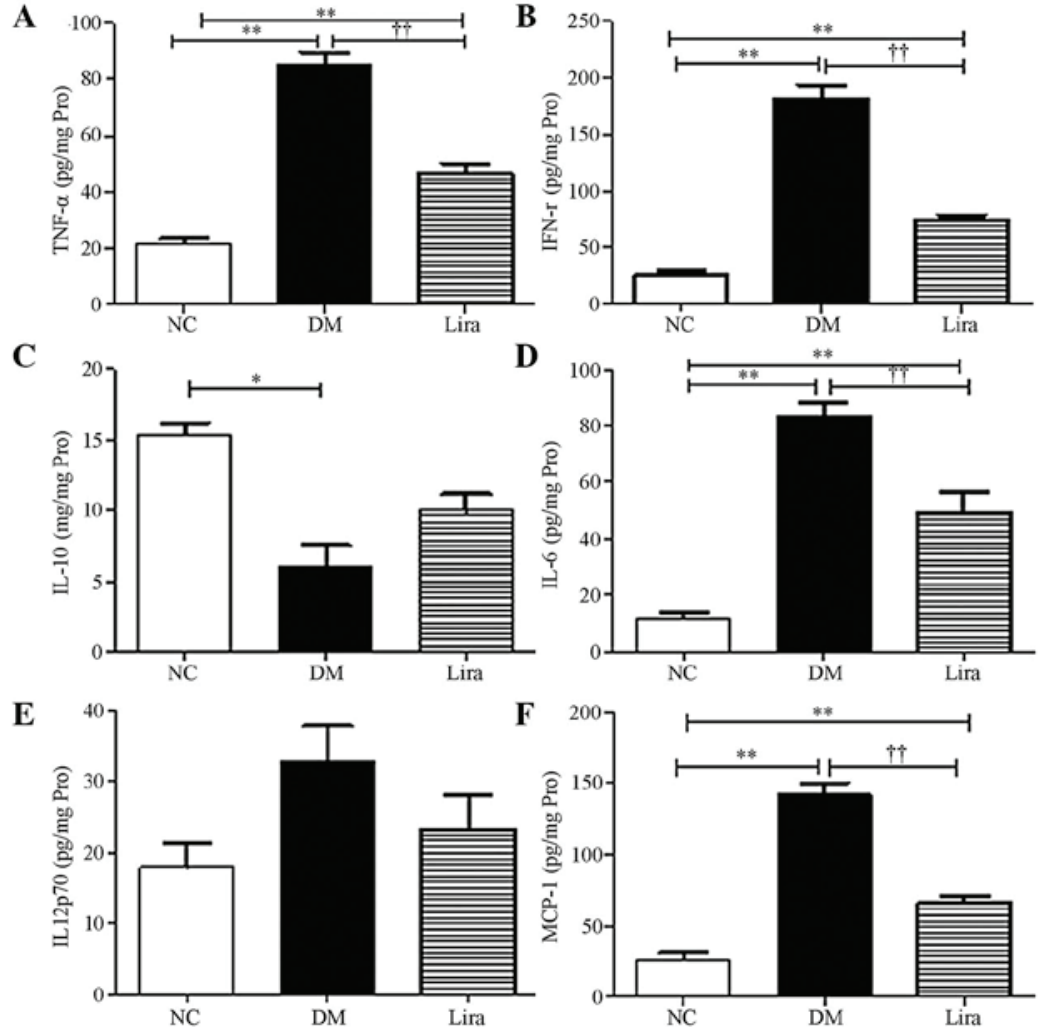

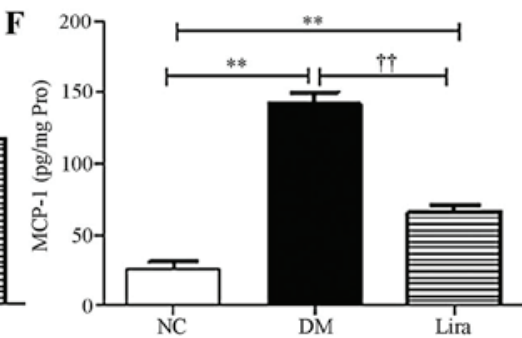

Figure 4. Liragluide downregulated inflammation in diabetic kidney. The protein expression levels of (A) TNF- $\alpha$, (B) IFN- $\gamma$, (C) IL-10, (D) IL-6, (E) IL-12p70 and (F) MCP-1 in diabetic kidneys. Renal tissue was homogenized and the concentrations of inflammatory cytokines were evaluated by an cytometric bead array kit. Values are expressed as the mean \pm standard deviation of the data. ${ }^{*} \mathrm{P}<0.05$ and ${ }^{* *} \mathrm{P}<0.01 \mathrm{vs}$. the control; ${ }^{\dagger} \mathrm{P}<0.05$ and $\dagger \dagger \mathrm{P}<0.01 \mathrm{vs}$. $\mathrm{DM}$. DM, diabetes mellitus rats induced by injection of streptozotocin; Lira, diabetic rats treated by injection liragrutide; TNF- $\alpha$, tumor necrosis factor- $\alpha$; IFN- $\gamma$, interferon- $\gamma$; IL, interleukin.

A

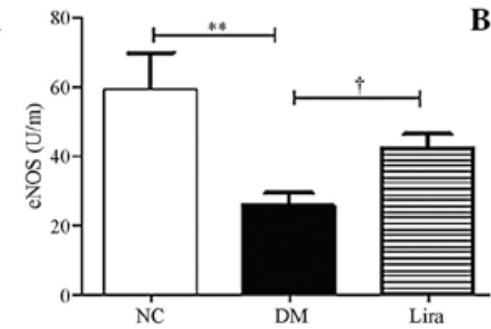

B

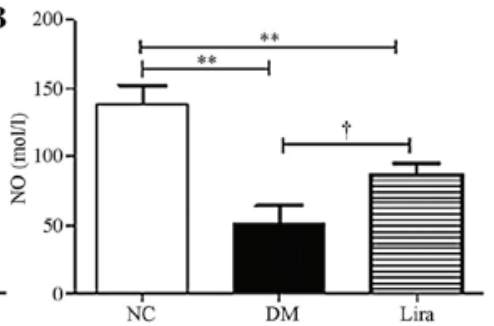

Figure 5. Liraglutide restored the activity of eNOS and the production of NO. (A) the activity of eNOS in kidneys; (B) the production of NO by the kidneys. eNOS activity classifying kit and NO test kit were used to detect the eNOS levels and NO in the glomerular, respectively. Values are expresssed as the mean \pm standard deviation of the data and demonstrate reduced NO production and decreased eNOS activity in the diabetic rat kidneys, and that Liragrutide restored eNOS activity and NO production. ${ }^{* *} \mathrm{P}<0.01$ vs. the control; ${ }^{\dagger} \mathrm{P}<0.05, \mathrm{P}<0.01 \mathrm{vs}$. DM. DM, diabetes mellitus rats induced by injection of streptozotocin; Lira, diabetic rats treated by injection liragrutide; eNOS, endothelial nitric oxide synthase; NO, nitric oxide.

Liraglutide improves eNOS activity and NO production.eNOS activity and glomerular NO synthesis were examined. Fig. 5 demonstrated that eNOS activity and NO production were decreased significantly in both diabetic groups. Liraglutide restored the eNOS activity $(\mathrm{P}<0.01$ or $\mathrm{P}<0.05)$ and $\mathrm{NO}$ production $(\mathrm{P}<0.01$; Fig. $5 \mathrm{~A}$ and $\mathrm{B})$.

\section{Discussion}

Liraglutide, a GLP-1 analogue, is a novel prospective medication for Type 2 diabetes mellitus. Liraglutide also facilitates the maintenance of glucose homeostasis by stimulating insulin secretion and inhibiting glucagon release in a glucose-dependent manner (13). Accumulating evidence indicates that GLP-1 analogues ameliorate diabetic nephropathy in mice and humans $(6,9,14)$; however, the underlying mechanism has remained elusive. In the present study, liraglutide activated eNOS activity in diabetic kidneys via downregulation of the NF- $\mathrm{KB}$ signaling pathway and reduction of chronic inflammation in diabetic rats. Therefore, liraglutide exhibits a protective function in diabetic kidneys. The results of the present study revealed that liraglutide significantly decreased inflammatory cell infiltration in the intestines, alleviated the damage of the glomerular filter barrier in diabetic rats and reduced urine volume, BUN, Scr and 24-h urine microalbumin levels. This result indicated that liraglutide elicits a protective effect 
on tissues against diabetic nephropathy in rats. The present study also identified that liraglutide significantly delayed hyperfiltration as indicated by the kidney weight index and reduced urinary protein excretion in diabetic rats (Table II). However, no significant difference was observed in the BG levels among the groups, which is consistent with results of previous studies $(9,10)$. The results further revealed that the protective effect of liraglutide against diabetic nephropathy in rats was independent of the glucose-lowering effect, but may be associated with the anti-inflammatory effect.

To identify the potential mechanism, the effect of liraglutide on the expression and activity of eNOS in the kidneys of diabetic rats was further assessed. No significant decrease was observed in the eNOS expression in mRNA and protein levels in the kidneys of diabetic rats. By comparison, eNOS phosphorylation on serine 1177 was increased, which is a process that is the key phosphorylation involved in eNOS activation (15). The decreased activity of eNOS in glomerular endothelial cells is the leading cause of NO secretion disorder (2). The present study also determined the NO production and the results demonstrated that liraglutide restored NO production. These results indicated that the dysfunction of eNOS in DN mainly occurred due to decreases in eNOS phosphorylation, further confirming the conclusions presented in previous studies $(2,3)$. Liraglutide also contributed to the pathogenesis of DN by increasing eNOS phosphorylation on serine 1177 and promoting NO production. Therefore, eNOS is a potentially new target for the treatment of diabetic nephropathy. Further studies are required to determine the mechanism by which eNOS activity is enhanced.

The present study further investigated the potential mechanism by which liraglutide improves eNOS activity. In this type of activity, chronic inflammation is important in the process of DN. Previous studies demonstrated that liraglutide suppressed inflammatory cytokine expression in diabetic patients $(7,16)$. This evidently indicated that liraglutide activates eNOS by downregulating chronic inflammatory pathways. NF- $\mathrm{B}$ is one of the key molecules that regulate chronic inflammation. The activation of NF- $\kappa \mathrm{B}$ and the transcription of certain pro-inflammatory chemokines, including MCP-1, TNF- $\alpha$ and intracellular adhesion molecule 1, are the markers of progressive diabetic nephropathy in patients with type 2 diabetes (17). By comparison, the inhibition of NF- $\kappa \mathrm{B}$ activity reduces the damage of diabetic nephropathy (6). These studies have markedly suggested that NF- $\kappa \mathrm{B}$ participates in the pathogenesis of diabetic nephropathy. The results also demonstrated that p65 expression and phosphorylation on serine 276 were increased in the kidneys of diabetic rats, which is an increase that is essential for NF- $\mathrm{B}$ p65-dependent cellular responses (18). The detection of $\mathrm{NF}-\kappa \mathrm{B}$ and the phosphorylated p65 subunit of NF- $\mathrm{B}$ is an effective method for examining $N F-\kappa B$ activation $(19,20)$. The levels of inflammatory cytokines downstream of $\mathrm{NF}-\kappa \mathrm{B}$ were further investigated in the present study. The results revealed that MCP-1, TNF- $\alpha$ and IFN- $\gamma$ were overexpressed in the kidneys of diabetic rats, which is a result that is consistent with that demonstrated in previous studies $(17,21)$. Liraglutide inhibited the increases in inflammatory cytokines, including MCP-1, TNF- $\alpha$, IFN- $\gamma$ and IL-6. Furthermore, it also restored the anti-inflammatory cytokine IL-10 levels; however, this difference was not statistically significant. The results indicated that the $\mathrm{NF}-\kappa \mathrm{B}$ signaling pathway is activated in diabetic nephropathy and liraglutide is able to suppress p65 expression and phosphorylation on serine 276 of $N F-\kappa B$. Liraglutide may further decrease the expression of inflammatory cytokines of kidneys in diabetic rats. Therefore, it is concluded that liraglutide elicits a protective effect on diabetic nephropathy by downregulating the $\mathrm{NF}-\kappa \mathrm{B}$ signaling pathway.

To the best of our knowledge, the present study was the first to identify that liraglutide activates eNOS activity via the $\mathrm{NF}-\kappa \mathrm{B}$ inflammatory pathway and elicits a protective effect on diabetic kidneys. However, the mechanism by which the $\mathrm{NF}-\kappa \mathrm{B}$ pathway regulates eNOS activity in diabetic nephropathy requires further study.

In conclusion, liraglutide may have a direct beneficial effect on eNOS activity and diabetic nephropathy by downregulating $\mathrm{NF}-\kappa \mathrm{B}$ inflammatory pathway without eliciting a glucose-lowering effect.

\section{References}

1. Nakagawa T: Is endothelial dysfunction more deleterious than podocyte injury in diabetic nephropathy? Kidney Int 83: 1202-1203, 2013

2. Nakagawa T, Sato W, Sautin YY, et al: Uncoupling of vascular endothelial growth factor with nitric oxide as a mechanism for diabetic vasculopathy. J Am Soc Nephrol 17: 736-745, 2006.

3. Nakagawa T, Sato W, Glushakova O, et al: Diabetic endothelial nitric oxide synthase knockout mice develop advanced diabetic nephropathy. J Am Soc Nephrol 18: 539-550, 2007.

4. Tian S, Gan Y, Li J, et al: Imbalance of glomerular VEGF-NO axis in diabetic rats: prevention by chronic therapy with propyl gallate. J Nephrol 24: 499-506, 2011.

5. Chiu WC, Chiou TJ and Chiang AN: $\beta$-Glycoprotein I inhibits endothelial cell migration through the nuclear factor $\kappa \mathrm{B}$ signalling pathway and endothelial nitric oxide synthase activation. Biochem J 445: 125-133, 2012.

6. Kim JE, Lee MH, Nam DH, et al: Celastrol, an NF- $\kappa \mathrm{B}$ inhibitor, improves insulin resistance and attenuates renal injury in $\mathrm{db} / \mathrm{db}$ mice. PLoS One 8: e62068, 2013.

7. Shiraki A, Oyama J, Komoda H, et al: The glucagon-like peptide 1 analog liraglutide reduces TNF- $\alpha$-induced oxidative stress and inflammation in endothelial cells. Atherosclerosis 221: 375-382, 2012.

8. Parthsarathy $\mathrm{V}$ and Hölscher $\mathrm{C}$ : The type 2 diabetes drug liraglutide reduces chronic inflammation induced by irradiation in the mouse brain. Eur J Pharmacol 700: 42-50, 2013.

9. Hendarto H, Inoguchi T, Maeda Y, et al: GLP-1 analog liraglutide protects against oxidative stress and albuminuria in streptozotocin-induced diabetic rats via protein kinase A-mediated inhibition of renal $\mathrm{NAD}(\mathrm{P}) \mathrm{H}$ oxidases. Metabolism 61: 1422-1434, 2012.

10. Sturis J, Gotfredse CF, Rømer J, et al: GLP-1 derivative liraglutide in rats with $\beta$-cell deficiencies: influence of metabolic state on $\beta$-cell mass dynamics. British J Pharmacol 140: 123-132, 2003.

11. Shiju TM, Rajesh NG and Viswanathan P: Renoprotective effect of aged garlic extract in streptozotocin-induced diabetic rats. Indian J Pharmacol 45: 18-23, 2013.

12. Hill HR and Martins TB: The flow cytometric analysis of cytokines using multi-analyte fluorescence microarray technology. Methods 38: 312-316, 2006.

13. Rigato $M$ and Fadini GP: Comparative effectiveness of liraglutide in the treatment of type 2 diabetes. Diabetes Metab Syndr Obes 7: 107-120, 2014.

14. Vretenar J, Jindal K and Senior PA: Effect of liraglutide on metabolic and renal outcomes over 12 months in community diabetic nephropathy clinics. Can J Diabetes O 37S4: S25, 2013.

15. García C, Nuñez-Anita RE, Thebault S, et al: Requirement of phosphorylatable endothelial nitric oxide synthase at Ser-1177 for vasoinhibin-mediated inhibition of endothelial cell migration and proliferation in vitro. Endocrine 45: 263-270, 2013. 
16. Parthsarathy $\mathrm{V}$ and Hölscher $\mathrm{C}$ : The type 2 diabetes drug liraglutide reduces chronic inflammation induced by irradiation in the mouse brain. Eur J Pharmacol 700: 42-50, 2013.

17. Xie X, Lan T, Chang X, et al: Connexin43 mediates NF- $\kappa \mathrm{B}$ signalling activation induced by high glucose in GMCs: involvement of c-Src. Cell Commun Signal 11: 38, 2013

18. Okazaki T, Sakon S, Sasazuki T, et al: Phosphorylation of serine 276 is essential for p65 NF-kappaB subunit-dependent cellular responses. Biochem Biophys Res Commun 300: 807-812, 2003.
19. Wang M, Liu R, Jia X, Mu S and Xie R: N-acetyl-seryl-aspartyllysyl-proline attenuates renal inflammation and tubulointerstitial fibrosis in rats. Int J Mol Med 26: 795-801, 2010

20. Jain SK, Velusamy T, Croad JL, Rains JL and Bull R: L-cysteine supplementation lowers blood glucose, glycated hemoglobin, CRP, MCP-1, and oxidative stress and inhibits NF-kappaB activation in the livers of Zucker diabetic rats. Free Radical Bio Med 46: 1633-1638, 2009.

21. Wada $\mathbf{J}$ and Makino $\mathrm{H}$ : Inflammation and the pathogenesis of diabetic nephropathy. Clin Sci (Lond) 124: 139-152, 2013. 